\title{
Multiple Labeling of Peptides via Orthogonal Coupling Reactions and Its Applications
}

\author{
Bikash Manandhar, Tae-Kyung Lee, and Jung-Mo Ahn* \\ Dept. of Chemistry and Biochemistry, University of Texas at Dallas, Richardson, TX, 75080, USA
}

\section{Introduction}

Labeling peptides have been widely practiced in numerous applications in chemistry and biology. For example, fluorescent dyes make peptides useful probes for in vitro and in vivo imaging and suitable substrates for determining enzyme activity; biotinylation has vast applications including affinity-based protein purification; PEGylation enhances plasma half-life and protects against metabolic degradation; radiolabeling offers in vitro and in vivo molecular imaging agents and targeted radionuclide therapy; cell permeable peptides give access to intracellular targets. Thus, peptides bearing such multiple labels may serve as powerful research tools and become handy for diverse biomedical studies. For labeling peptides, a number of coupling methods have been reported, such as alkyne-azide click chemistry, thiol-maleimide coupling, Staudinger ligation, and oxime/hydrazone formation. Despite the versatility and broad use in traditional organic reactions, Suzuki-Miyaura coupling has been rarely practiced in peptide labeling. In addition, while each of these methods has been efficiently used alone in peptide conjugation, their combination for orthogonal coupling reactions was not well studied.

\section{Results and Discussion}

Previously, we examined and optimized the Suzuki-Miyaura cross-coupling for peptide ligation by surveying various catalysts, bases, temperature, solvents, and additives [1]. We also determined the effects of amino acid side chains on the ligation reactions of peptides. In order to demonstrate its orthogonality towards widely used thiol-maleimide chemistry and copper-catalyzed azide-alkyne cycloaddition (CuAAC), peptide $\mathbf{1}$ bearing three different functional groups (a phenyl boronic acid, a sulphydryl group, and an azide) was designed and synthesized. Since a thiol is not compatible to Suzuki reactions, it was kept protected with a tBu group. We also prepared a cell penetrating poly-lysine peptide with iodobenzoate (2); an acetylene-conjugated biotin (3); and a maleimide-functionalized fluorescein (4) (Figure 1). Peptides 1 and 2 were synthesized in solid-phase by following standard Fmoc- ${ }^{\mathrm{B}} \mathrm{Bu}$ protocol. The resulting peptides were cleaved from resin with TFA and purified by RPHPLC.

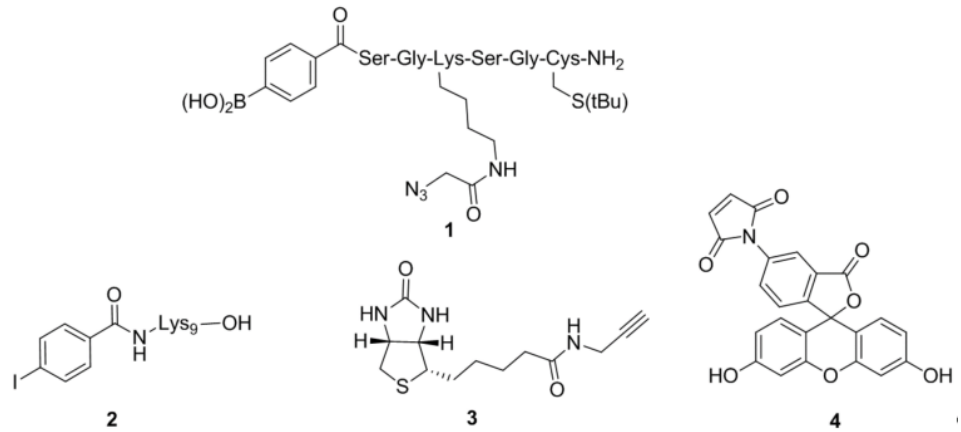

Fig. 1. Structures of reacting components used for multiple labeling.

Consecutive labeling of peptide 1 was carried out with poly-lysine 2, biotin 3 and fluorescein 4 via Suzuki-Miyaura cross-coupling, CuAAC, and thiol-maleimide methods, respectively (Scheme 1). The conditions and reagents of these coupling reactions were found to be compatible and orthogonal to each other as indicated by a high purity of the products analyzed by HPLC. The multiply labeled peptide 7 was incubated with HEK293 cells for 12 hours in an attempt to demonstrate the utility of multiple labeling. Peptide 7 was carried into the cells by the conjugated cell penetrating poly-lysine peptide moiety, which was easily visualized with fluorescence microscope owing to the fluorescein moiety (Figure 2). 


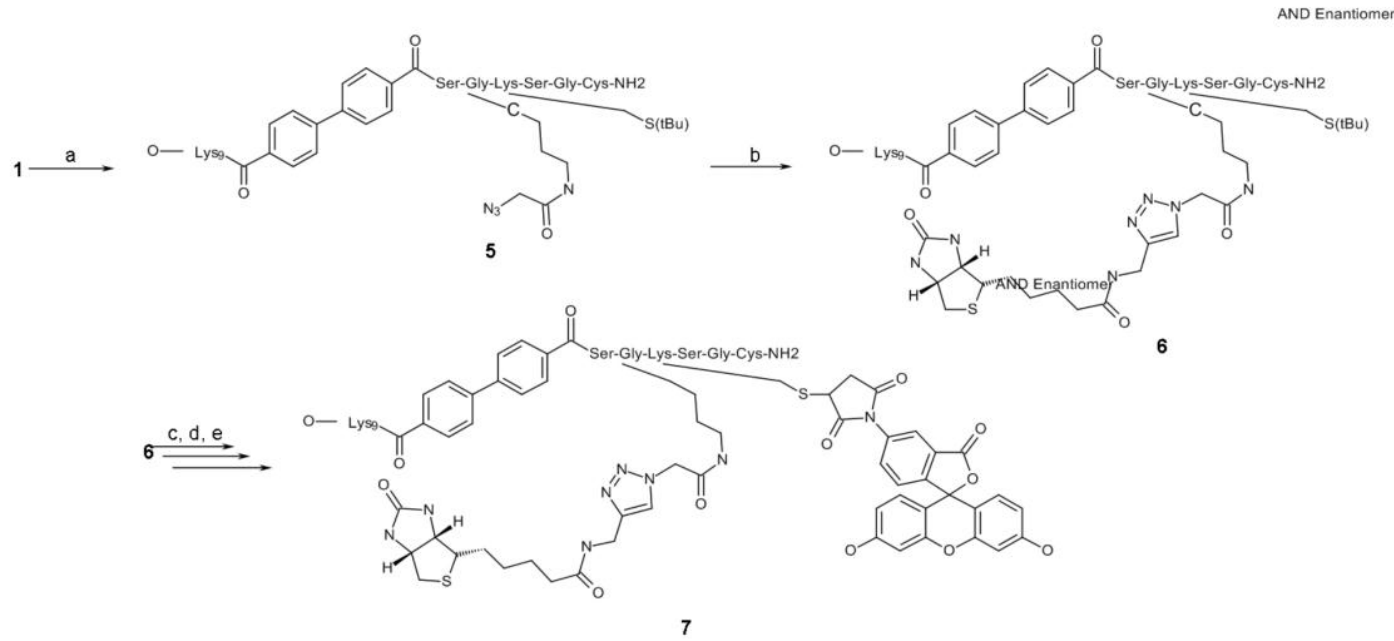

Scheme 1. Multiple labeling of peptides. (a) 2, $\mathrm{Pd}(\mathrm{dppf}) \mathrm{Cl}_{2}, \mathrm{~K}_{2} \mathrm{CO}_{3}, \mathrm{DMF}, \mathrm{H}_{2} \mathrm{O}$; (b) 3, $\mathrm{CuSO}_{4}, \mathrm{NaAsc}$, $\mathrm{DMF}, \mathrm{tBuOH}, \mathrm{H}_{2} \mathrm{O}$; (c) DTNP, TFA; (d) TCEP, $\mathrm{H}_{2} \mathrm{O}$; (e) 4, PBS.

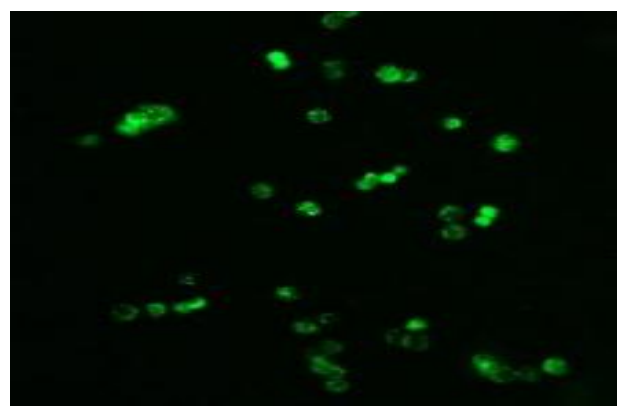

Fig. 2. Fluorescence microscope image of HEK293 cells after $12 \mathrm{~h}$ incubation with peptide 7.

In summary, we have developed an efficient synthetic strategy for multiple labeling of peptides via various orthogonal coupling reactions, such as Suzuki-Miyaura cross-coupling, thiol-maleimide reaction, and alkyne-azide cycloaddition. The potential of these orthogonal chemistries was demonstrated by a peptide with multiple labels of a cell penetrating peptide, a fluorescent dye, and a biotin.

\section{Acknowledgments}

This work was supported in part by the Welch Foundation (AT-1595), Juvenile Diabetes Research Foundation (37-2011-20), and Cancer Prevention and Research Institute of Texas (RP120717-P4).

\section{References}

1. Lee, T.-K. Manandhar, B. and Ahn, J.-M., in Lebl, M. (Ed.) Peptides: Peptides Across the Pacific (Proceedings of the 23rd American Peptide Symposium), American Peptide Society, San Diego, 2013, p. 234, http://dx.doi.org/10.17952/23APS.2013.234 